\section{Review: antibiotic treatment for 7-14 days reduces treatment faillure in children with urinary tract infection}

\author{
Keren R, Chan E. A meta-analysis of randomized, controlled trials comparing short-and long-course antibiotic therapy \\ for urinary tract infections in children. Pediatrics 2002;109:e70. \\ QUESTION: In children with urinary tract infection (UTI), is a long course (LC) of \\ antibiotic treatment more effective than a short course (SC) for preventing treatment \\ failure or reinfection?
}

\section{Data sources}

Studies were identified by searching Medline and the Cochrane Library (all up to April 2001), reviewing bibliographies of relevant articles, and contacting experts in the field for any other published or unpublished studies.

\section{Study selection}

Studies published in English were selected if they were randomised controlled trials (RCTs) comparing SC with LC outpatient antibiotic treatment for acute UTI in children $0-18$ years of age. Studies that were restricted to children with recurrent UTI or that included children with asymptomatic bacteriuria were excluded from the review.

\section{Data extraction}

2 reviewers independently extracted data on setting, sample size, study quality, patient demographics, details of the intervention, definition of UTI, attempt to distinguish lower from upper UTI, and outcomes. Main outcomes included treatment failure (persistent infection or relapse) and reinfection (initial bacteriological cure followed by recurrence of symptoms and infection with a different organism).

\section{Main results}

13 RCTs ( $\mathrm{n}=924)$ that evaluated 14 antibiotics were included in the meta-analyses. Comparisons included LC $(7-14 \mathrm{~d}) \cup \mathrm{SC}(1 \mathrm{~d}, 3 \mathrm{~d}$, or single dose [SD]) treatment (12 RCTs), LC $v$ SD or 1 day treatment (7 RCTs), and LC $v 3$ day treatment (5 RCTs). Metaanalyses were done using a random effects model. Metaanalysis of all studies, and of RCTs restricted to children with lower UTIs, showed a lower rate of treatment failure in the LC group than in the SC group; the groups did not differ for reinfection rates (table). Subgroup analysis of studies comparing LC with SD or 1 day treatment also showed a lower rate of treatment failure in the LC group than the SC group; the groups did not differ for reinfection rates (table). Subgroup analysis of studies comparing LC with 3 day treatment showed no difference between the 2 groups for treatment failure or reinfection (table)

\section{Conclusions}

In children with urinary tract infection, a long course of outpatient treatment with antibiotics $(7-14 \mathrm{~d})$ is more effective than a short course ( $\leq 3 \mathrm{~d})$ for preventing treatment failure. Both regimens have similar reinfection rates. Long course $(L C)(7-14 d)$ v short course $(S C)(1$ d or 3 d, or a single dose [SD]) antibiotic
treatment for urinary tract infection in children at 1 to 10 days*

\begin{tabular}{clllll} 
Outcomes & NRCTs & Comparison & $\begin{array}{l}\text { Weighted } \\
\text { event rates }\end{array}$ & RRR ( $95 \%$ Cl) & NNT (CI) \\
\hline TF & 12 & LC $v$ SC (overall) & $9 \% v 16 \%$ & $48 \%(18$ to 67$)$ & 14 (8 to 61$)$ \\
\hline 10 & LC $v$ SC (PLUTI only) & $11 \% v 16 \%$ & $42 \%(8$ to 64$)$ & 18 (9 to 203$)$ \\
\hline 7 & LC $v$ SD or 1 day & $11 \% v 25 \%$ & $61 \%(28$ to 79$)$ & 7 (5 to 15$)$ \\
\hline Reinfection & 5 & LC $v 3$ day & $9 \% v 12 \%$ & $26 \%(-42$ to 62$)$ & Not significant \\
\hline & LC $v 3$ day & $7 \% v 8 \%$ & $9 \%(-110$ to 61$)$ & Not significant \\
\hline 6 & LC $v$ SC (overall) & $10 \% v 8 \%$ & $28 \%(-36$ to 156$)$ & Not significant \\
\hline 5 & LC $v$ SC (PLUTI only) & $14 \% v 10 \%$ & $39 \%(-38$ to 211$)$ & Not significant \\
\hline 2 & LC $v$ SD or 1 day & $25 \% v 8 \%$ & $165 \%(-10$ to 681$)$ & Not significant \\
\hline
\end{tabular}

${ }^{*} \mathrm{NRCTs}=$ number of randomised controlled trials; TF $=$ treatment failure; PLUTI = patients with lower urinary tract infection. Other abbreviations defined in glossary; RRR, RRI, NNT, NNH, Cl, and weighted event rates calculated from data supplied by author using a random effects model.

\section{COMMENTARY}

The review by Keren and Chan concludes that clinicians should continue to treat children with UTI with antibiotics for 7-14 days (LC) and that no additional comparative trials are warranted until more accurate methods are identified for distinguishing upper from lower UTI in children.

Strengths of this review include attempts to identify unpublished studies, quality scoring of the studies, and use of a random effects model for meta-analysis. Limitations include restriction of the search to Medline and to studies published in English and failure to consider choice of antibiotic as a source of heterogeneity.

Although this review shows that LC treatment is more effective than SD or 1 day treatment, it does not show that LC treatment is more effective than 3 day treatment. The authors acknowledge that this might be attributable to a small sample size. However, it is curious that they then suggest that no additional comparative studies are warranted. In fact, research to date has not ruled out the possibility that 3 day treatment is as effective as LC treatment.

Further complicating this review is the large number of different antibiotics evaluated. Review of the individual studies in table 1 of the original article suggests that certain SC antibiotics may indeed be effective, and perhaps by pooling results from studies of all drugs, these effects were lost. This is particularly important given that the 2 factors the authors chose to examine to explain heterogeneity among studies (age and study quality) failed to do so.

What does all this mean to the clinician? Firstly, it is advisable to continue with conventional LC antibiotic treatment (7-14 d) for children with UTI. As recommended by the authors of previous reviews on this topic, further studies are needed to compare 3 day treatment with LC treatment and to look more closely at specific antibiotics. ${ }^{2}$ Judith Daniels, RN, MSN Instructor, College of Nursing
University of Kentucky, Lexington, Kentucky, USA
Alba DiCenso, RN, PhD
Professor, School of Nursing
McMaster University, Hamilton, Ontario, Canada

1 Tran D, Muchant DG, Aronoff SC. Short-course versus conventional length antimicrobial therapy for uncomplicated lower urinary tract infections in children: a meta-analysis of 1279 patients. J Pediatr 2001;139:93-9.
Khan AJ. Efficacy of single-dose therapy of urinary tract infection in infants and children: a review. J Natl Med Assoc 1994;86:690-6. 